\title{
Corela
}

Cognition, représentation, langage

1-1 | 2003

Vol. $1, \mathrm{n}^{\circ} 1$

\section{When et la hiérarchie propositionnelle}

\section{Emmanuelle Roussel}

\section{OpenEdition}

\section{Journals}

Édition électronique

URL : http://journals.openedition.org/corela/649

DOI : $10.4000 /$ corela. 649

ISSN : 1638-573X

\section{Éditeur}

Cercle linguistique du Centre et de l'Ouest - CerLICO

\section{Référence électronique}

Emmanuelle Roussel, «When et la hiérarchie propositionnelle », Corela [En ligne], 1-1 | 2003, mis en ligne le 01 avril 2003, consulté le 01 mai 2019. URL : http://journals.openedition.org/corela/649; DOI : $10.4000 /$ corela.649

Ce document a été généré automatiquement le 1 mai 2019.

\section{(c) (i) (2)(2)}

Corela - cognition, représentation, langage est mis à disposition selon les termes de la licence Creative Commons Attribution - Pas d'Utilisation Commerciale - Partage dans les Mêmes Conditions 4.0 International. 


\title{
When et la hiérarchie propositionnelle
}

\author{
Emmanuelle Roussel
}

\section{À Rosalind Dilys}

1 Nous connaissons tous when, opérateur privilégié de l'expression de relations temporelles. L'opérateur et son fonctionnement ont fait l'objet de nombreux articles, dans lesquels les diverses valeurs de when mises à jour le sont, en règle générale, d'un type d'énoncé à un autre. La particularité de when y est fort bien décrite et explicitée. On apprend que when est un opérateur "à sémantisme variable" selon le contexte où il apparait. Chaque énoncé est susceptible de générer une valeur de when qu'il n'aura pas dans un autre ${ }^{1}$. When offre néanmoins d'autres interprétations possibles, comme le laisse entendre G. Deléchelle: "[...] on peut se demander s'il ne faut pas poser pour certaines constructions concomitantes un statut tendant vers la coordination"2.

2 Je souhaiterais, dans cet article, réfléchir à ce phénomène et montrer par là même que when peut tout à fait conserver ses propriétés "plurielles" au sein, une fois n'est pas coutume, d'un seul et même énoncé. Ainsi She had been healthy and beautiful when he married her, énoncé sur lequel j'ai auparavant longuement travaillé3. C'est, je le souligne, par choix que j'ai entrepris d'étudier cet énoncé de manière isolée, les relations qu'il met en place étant suffisamment intéressantes pour être matière à réflexion. Un seul énoncé peut en effet conduire à autant d'analyses captivantes et ouvrir autant de voies de recherche que tout un corpus.

3 Cet énoncé, concernant le sémantisme profond de when, n'entre dans aucune catégorie répertoriée jusqu'ici. Et pourtant, il pose essentiellement trois questions : celle de son sémantisme même, celle du statut de la proposition subordonnée temporelle et celle du comportement de when dans cet exemple précis. Nous pouvons, d'emblée, voire que la temporelle constitue le pivot de la problématique, car son introduction en modifie les données. 
De fait, sans la subordonnée, she had been healthy and beautiful peut prendre deux valeurs étant donné le caractère implicite de tout repère extérieur à la relation $\mathrm{S} / \mathrm{P}$. Ces deux valeurs sont directement liées aux propriétés du perfect sur le verbe. D'une part, l'énoncé renverra à une continuité référentielle avec $\mathrm{T}_{1}^{(4)}$ si la relation $\mathrm{S} / \mathrm{P}$ [she - be healthy and beautiful] se poursuit réellement jusqu'en $\mathrm{T}_{1}$, est toujours vraie en $\mathrm{T}_{1}$. D'autre part, le même énoncé traduira une rupture référentielle couplée à une continuité énonciative si [she - be healthy and beautiful], aussi révolue soit-elle, est appréciée dans son rapport énonciatif à $\mathrm{T}_{1}$. Seul le contexte permettra de sélectionner telle ou telle valeur en fonction de ce que le lecteur / co-énonciateur sait de she notamment. ${ }^{5}$ Dès lors que when he married her est introduit, l'énoncé prend une troisième signification, celle d'une continuité référentielle suivie d'une rupture, que l'on schématisera ainsi :

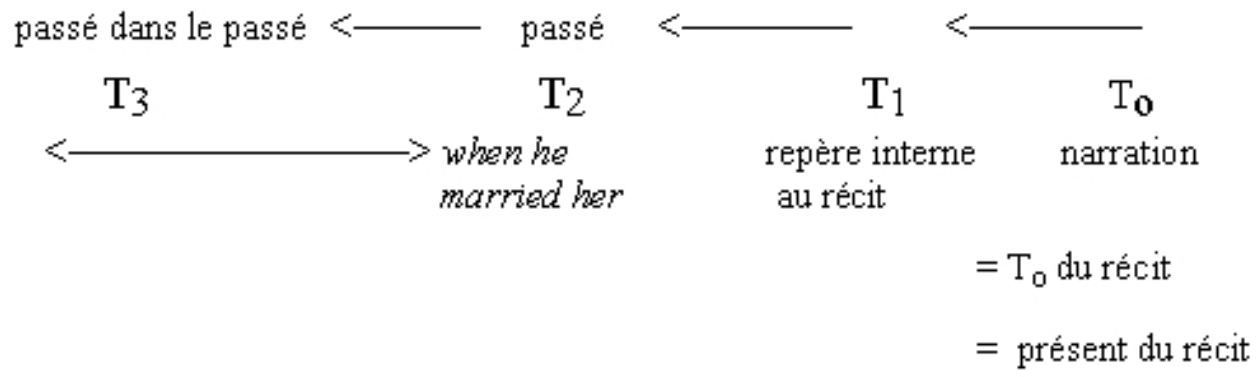

5 La continuité référentielle est vraie entre $T_{3}$ et $T_{2}$ (celle-ci peut être définitive ou constituée de plusieurs instances de la validation de S/P), puis la rupture s'instaure automatiquement entre $T_{2}$ et $T_{1}$. L'apparition de la proposition subordonnée, en important un nouveau repère, bouleverse toute la relation initiale de repérage et redistribue les rôles entre les différents jalons temporels.

6 Qu'est-ce qui permet ainsi à cette subordonnée de modifier la signification d'un énoncé original ? Tire-t-elle cette possibilité des propriétés de ses constituants internes ou de sa mise en contact avec la principale ? Est-elle nouvelle ou présupposée ? Qu'est-ce qui fait que son introduction puisse changer aussi radicalement le sémantisme de l'énoncé ?

7 Je propose de réfléchir à la question en suivant principalement deux directions. La première, classique, à base de manipulations contrastives. La seconde, personnelle, va bien au-delà.

\section{When, opérateur unique}

8 Dans cette optique, nous partons du principe que le sémantisme de l'énoncé est imputable soit à when, soit à son interprétation ou encore à l'aspect interne des lexèmes verbaux en jeu. Il se trouve par exemple que be, verbe d'état de la proposition matrice, est plus que tout autre à même de signifier la continuité et contribue lui aussi à fixer un intervalle. En revanche, marry dénote lui un procès à bornes confondues (ou procès ponctuel) dans lequel le début implique la fin. Le point de vue qui lui est associé est quantitatif : il s'agit d'une occurrence particulière. L'opposition entre ces deux verbes concourt au contraste perçu à leur lecture.

9 Toujours en ce qui concerne les types de procès, nous noterons cependant que contrairement à ce qui semble généralement être le cas dans une telle collocation, (past perfect / prétérit) où les deux procès sont consécutifs, il n'y a pas dans notre exemple de possible construction d'un quelconque intervalle entre les deux procès mentionnés ${ }^{6}$. 
10 Nous pouvons alors ensuite, comme il est d'usage, travailler l'inversion des deux segments étudiés en cherchant à savoir quelles sont les répercussions sur le sémantisme de l'énoncé :

(1a) When he married her, she had been healthy and beautiful.

11 Le sens profond de (1a) est similaire à celui de (1). Avec l'antéposition de la proposition subordonnée temporelle, se sont prioritairement les effets de sens portés par le prétérit qui sont perçus. L'énoncé est alors vu comme plus ponctuel, définitif, axé sur les circonstances temporelles de validation de la relation prédicative [he - marry her] plutôt que sur la proposition principale. Le message est également plus objectif, l'énoncé est de l'ordre du constat détaché.

12 Il reste que (1a) n'est pas naturel. On lui préférerait sans aucun doute (1b), avec la principale au prétérit :

(1b) When he married her, she was healthy and beautiful. Mais alors, when traduit une relation de concomitance, absente de (1).

13 A première vue donc, (1), avec sa valeur propre, n'est possible que dans l'ordre donné.

14 Qu'en est-t-il exactement de ce rapprochement de when avec as, ou même avec after, lesquels sont fréquemment présentés comme ses substituts privilégiés? Etant entendu que as, opérateur d'identification, traduit une relation de concomitance (dans son emploi temporel), et que after, opérateur de différenciation, instaure quant à lui une relation de consécution entre $\mathrm{P}_{1}$ et $\mathrm{P}_{2}$, l'occurrence de when de (1) peut-elle se construire avec as ou after? Je pense que non, essentiellement dans la mesure où (1) illustre un emploi de when où il marque une interception à un moment $\mathrm{x}$ sans création d'un quelconque écart entre les deux procès (ce qui est précisément le rôle de after) :

(1c)? She had been healthy and beautiful as he married her.

(1c')? As he married her she had been healthy and beautiful ${ }^{7}$.

15 S'ils étaient acceptables, et avec une lecture temporelle donc, ces énoncés véhiculeraient une relation de concomitance (au sens de " simultanéité "). Ce qui ne conviendrait pas, en tout état de cause.

(1d) * She had been healthy and beautiful after he married her.

(1d')? After she had been healthy and beautiful, he married her.

(1d") She had ceased being healthy and beautiful after he married her.

Seul (1d") est grammatical et acceptable, mais dans un sens différent de (1).

17 La permutation de when avec as et after ne fonctionne donc pas. Ce qui tendrait à prouver que le sémantisme de (1) est bien imputable à when (entre autres), sinon d'autres marqueurs pourraient fort bien être utilisés à sa place. Mais la valeur de when dans (1) est a priori temporelle, et pourtant les tests (1c) et (1d) sont irrecevables...

18 A mon sens, cela est dû au fait qu'il n'est pas question, en (1), de poser une relation d' antériorité entre le procès au past perfect et celui au prétérit (ce qui serait le cas si after était acceptable). Il n'est pas non plus question de rendre compte d'une relation de cause à effet entre $\mathrm{P}_{1}$ et $\mathrm{P}_{2}$, qui n'est pourtant que la seule valeur que peut revêtir as pour pouvoir fonctionner dans notre énoncé. Mais (1) n'est pas causal. D'où la non validité d'une permutation totalement satisfaisante avec as.

As pose donc problème, qu'il soit temporel ou causal. En effet :

a) temporel,

- as requiert un même temps sur les deux prédicats [ce qui n'est, une fois encore, pas le cas pour (1)] et 
- ne peut introduire des verbes renvoyant à une propriété (or be healthy and beautiful est bien la prédication d'une propriété).

b) causal, il crée un hiatus, car la causalité est la métaphore de la consécution [or il n'y a pas de relation de consécution en (1)] ;

Il n'est enfin pas non plus question de recouvrement (total ou partiel) des deux procès, autre sémantisme régulièrement rencontré avec when ${ }^{8}$. L'impossibilité d'une telle lecture est notamment due ici à l'aspect lexical de <MARRY $>9$. Ce qui tend à renforcer le rôle de l'aspect interne des verbes.

When ne semble pas pouvoir ici permuter avec l'une ou l'autre de ses "doublures" préférentielles. Même pas avec before, qui indiquerait qu'au moment du mariage, la relation prédicative [she - be healthy and beautiful] n'est plus validée. Et pourtant elle l'est bien en (1).

Néanmoins, (1) fonctionne ! Je propose ici une lecture nouvelle du comportement de when

\section{When en contexte}

21 La seconde étape de ma réflexion s'appuie non plus exclusivement sur when mais également sur d'autres constituants de l'énoncé. Soit les trois manipulations suivantes :

(1) She had been healthy and beautiful when he married her.

(1e) She was healthy and beautiful when he married her.

(2) She is healthy and beautiful now.

(2a)* She has been healthy and beautiful now.

22 La seconde paire d'énoncés témoigne de l'impossibilité de conserver le perfect sur BE avec now. Nous abordons ici la problématique now / then, où then en est le terme second qui suppose une mise en place initiale en now. Dans ce duo, la première étape consiste en la mise en place du repère (now), la seconde est la possibilité d'introduire une rupture temporelle avec then ${ }^{10}$. Then vient en second, car il contient TH-, morphème témoin d'un travail supplémentaire d'identification. Celle-ci se trouve précisée et requiert par là même un contexte. Then, c'est not now, où la négation dit une relation à un moment autre de l'énonciation. TH- transcende $\mathrm{T}_{\mathrm{o}}$. Il est la trace, dans un énoncé de la forme de (1), d'une première mise en relation d'un sujet et d'un prédicat. Puisqu'il est nécessaire de la "montrer", cela signifie que cette relation est hors de la sphère d'élocution, donc distincte de now. Cette première liaison prédicative est par conséquent notionnelle avant tout, c'est une abstraction, dans la mesure où l'énonciateur saisit ce à quoi then réfère par " reconstruction" mentale. Then n'est en effet pas systématiquement couplé avec now dans les énoncés, et pourtant cela ne bloque jamais la compréhension que nous pouvons avoir du sémantisme de then.

23 La marque d'antériorité attachée à then est d'abord une antériorité intellectuelle, un passé de prédication, un acquis linguistique. Dès lors que then est associé à un marqueur de révolu, quel qu'il soit, l'antériorité linguistique dont il est porteur se fond en antériorité temporelle. Avec then, on est par conséquent dans l'anaphore.

Or, au présent (She is healthy and beautiful now), temps qui coïncide avec l'énonciation, on en est bien à la première étape. Ce dont il s'agit alors, c'est de fixer un repère, ce qui suppose une parfaite stabilité de ce dernier. Pour fixer, il faut un aoriste, à savoir un présent simple pour un verbe d'état. Ce présent simple devient alors une époque repère 
pouvant intervenir comme point de départ dans un nouveau repérage. Le recours à l'aoriste se justifie par le fait que si now constitue effectivement une base pour la suite, cette base doit pouvoir être reprise. Or on ne peut reprendre qu'un entier, à savoir un aoriste sur le plan temporel.

Parallèlement, la raison de l'incompatibilité entre now et le present perfect pour notre énoncé (*She has been healthy...now) pourrait être que le present perfect est une localisation sur le mode have. Have, c'est l'attribution caractérisante qui précède l'identité (qui n'est autre que be: identité constituée de la somme de toutes les caractéristiques). Avec have, on en est au stade de la mise en place de la caractéristique, on se trouve dans une phase antérieure à l'assertion de l'être (be) comme entité close.

Now, en tant que repère, requiert la stabilité, une unité temporelle précise, un aoriste temporel. Ce qui est, entre autres, à l'origine de la compatibilité entre now et be, c'est certainement la stabilité nécessaire au premier qu'il trouve dans l'être, l'unité, l'entité parfaitement circonscrite et pouvant être reprise comme telle, du second.

C'est la raison pour laquelle, dans mon exemple, now ne fonctionne pas avec le present perfect, forme qui traite du passé dans son rapport (référentiel ou énonciatif) au présent et à $\mathrm{T}_{0}$. Il est question là de translation entre les deux sphères temporelles. Ce terme de "translation" renvoie aux notions de mouvement et d'instabilité, c'est-à-dire à une réalité autre que l'entier statique. Or il s'avère que now ne peut se combiner qu'à l'identité.

\section{Rôle du contexte temporel}

Voici comment les choses se passent dans la sphère temporelle du présent. Le passage à celle du révolu offre au moins deux possibilités de conjugaison pour BE, had been et was. En outre, il apparait que cette double possibilité va de pair avec une modification sémantique de when, ou à tout le moins une modification de son comportement au regard de la principale.

Prenons She was healthy and beautiful when he married her, où BE est conjugué au prétérit simple.

Dans ce cas, nous nous apercevons que when introduit le repère [he - marry her]. Ce repère fait là fonction d'élément nouveau, de base sur laquelle va venir se construire toute la relation S/P [she - be healthy and beautiful]. Pour véritablement pouvoir fonctionner comme repère, nous avons vu qu'il faut que ce soit un aoriste. Puisque l'on évolue dans la sphère du révolu, cet aoriste est le prétérit, le temps de la narration considérée comme un acquis suffisamment stable pour qu'il puisse, à son tour, générer un univers stable. Dans cette analyse, [he - marry her] constitue le point de départ par rapport auquel se construit la relation $\mathrm{S} / \mathrm{P}$ de la matrice. Ce qui signifie que was healthy and beautiful et married her sont en relation "d'isoplanéité temporelle" : les deux segments ont la même référence temporelle. L'énoncé va les mettre sur le même plan, les joindre. Dans ce cas, when lie simplement le repère et le repéré. Il s'agit d'un emploi temporel pur où when est posé en toute objectivité. Considéré depuis le présent, l'énoncé illustre un seul décalage par rapport à la narration. Le tout est renvoyé en $\mathrm{T}_{1}$ de façon homogène. Ici, la proposition temporelle (le repère) me semble être neuve, donnée au moment de l'élaboration de l'énoncé ${ }^{11}$. Ce repère (de l'aoriste sur be) ne peut être qu'un élément nouveau, et non ancien. 
31 En revanche, dans She had been healthy and beautiful when he married her, avec be au past perfect, les choses se passent différemment. La forme composée sur BE indique implicitement que le repère [he - marry her] est la trace d'un premier ${ }^{12}$ repérage temporel dans le révolu (puisque had been se construit par rapport à $\mathrm{T}_{1}$ et non plus par rapport à $\mathrm{T}_{\mathrm{o}}$ ). Ce qui tendrait à signifier que la proposition subordonnée temporelle est ancienne en (1). En effet, le repère introduit par when contient une première référence temporelle, $\mathrm{T}_{1}$. En tant qu'aoriste, ce repère est fixe.

[She - be healthy and beautiful], le repéré, peut alors être analysé de deux manières différentes selon que l'on considère les repères en soi ou les relations qui s'établissent entre eux.

Soit il est seulement question d'établir un positionnement des repères, et l'énoncé traduit une hiérarchisation temporelle. Il met en place un nombre $\mathrm{x}$ de repères entre lesquels peuvent s'instaurer plusieurs repérages possibles (par exemple entre $T_{3}$ et $T_{0}, T_{3}$ et $T_{1}, T_{2}$ et $\mathrm{T}_{\mathrm{o}}$, etc.).

Soit ce qui importe ce sont les relations entre les repères, et alors deux relations sortent du lot, la continuité entre $T_{3}$ et $T_{2}$ et la rupture entre $T_{2}$ et $T_{1}$, la première se trouvant spontanément accentuée par l'établissement de la seconde. $\mathrm{T}_{2}$ apparaît alors comme un marqueur de contraste dans la mesure où il occasionne un changement de polarité. Le mariage, annoncé par [he - marry her] rompt effectivement la relation entre $\mathrm{T}_{3}$ et $\mathrm{T}_{2}$, nous on passons du positif au négatif. $E n \mathrm{~T}_{2}$, la relation $\mathrm{S} / \mathrm{P}$ [she - be healthy and beautiful] est interrompue. Nous observons que les formes employées sur les verbes be et marry témoignent de la chose. Had-en, le past perfect, rend compte d'un repérage dans le passé. Il fait la synthèse, via have ${ }^{13}$, de deux moments distincts du passé. En partant du principe que l'invariant du past perfect est l'expression d'un "prétérit de parfait" et que, plutôt que d'être désigné par had-en, il se schématiserait en ${ }^{(14)}$ :

\section{$\mathrm{S} \quad[$ HAVE $\quad$ Ved2 $]$ ed 3}

nous remarquons que nous avons deux étapes.

La première, [have $\mathrm{V}_{\mathrm{ed} 2}$ ] rend compte d'une localisation de type have présentée comme réalisée avant $T_{1}$. L'intégration du prédicat [be healthy...] à la sphère de she est alors obligatoirement antérieure à $\mathrm{T}_{1}$, et elle est déclenchée par when. $-\mathrm{Ed}_{2}$ (ou -en) souligne cette antériorité tandis que dans un second temps, - $\mathrm{ed}_{3}$ (ou le prétérit sur have) repère l'événement par rapport à [he - marry her], c'est-à-dire qu'il vient donner une nouvelle identité temporelle à [have $\left.\mathrm{V}_{\mathrm{ed} 2}\right]^{15}$.

La combinaison [have $\left.\mathrm{V}_{\text {ed } 2}\right]_{\mathrm{ed} 3}$ rapporte l'événement réalisé en $\mathrm{T}_{3}$ ([be healthy...]) à une autre réalisée en $\mathrm{T}_{2}$ (à savoir [marry her]).

37 Or, pour en revenir à mon exemple, le décalage évident entre $\mathrm{T}_{3}$ et $\mathrm{T}_{2}$ ne permet pas d'interpréter when comme conjonction temporelle d'isoplanéité. Puisque $\mathrm{T}_{2}$ est différent de $\mathrm{T}_{1}$, alors when ne peut que marquer un contraste. A ce titre, il est le signe de l'ouverture d'un nouvel événement lui-même source de rupture ([marry her]).

Et l'on se rend compte alors que (1) est une icône du sémantisme profond de when conjonction. Il reproduit la construction du sens même de l'opérateur. En effet, R. Declerck nous apprend (1997: 46) que de mot interrogatif, when est utilisé comme 
relatif à la fin du Vieil Anglais. Il peut alors avoir un antécédent nominal temporel, ou pas d'antécédent exprimé. Ce dernier cas d'emploi est celui que l'on retrouve aujourd'hui pour when conjonction. When y est par conséquent défini comme "adverbe à antécédent amalgamé", où ce qu'il amalgame sont des adverbiaux (d'où son emploi comme conjonction) : "then when". Formé à la fois sur then et when, when conjonction ne peut donc avoir qu'une double orientation, vers le préconstruit ( $\mathrm{(H-)}$ et vers la droite ( $\mathrm{WH}-$ ) pour le remplissage informationnel. Ce qui confirme si besoin était la raison pour laquelle mon énoncé pourrait fort bien se transformer en (3):

(3) She had been healthy and beautiful then.

(puisque TH-, trace d'un acquis de contenu prédicatif, est orienté vers la gauche) mais pas en (3a) :

(3a) * She had been healthy and beautiful when.

WH-, prospectif, indiquant l'attente d'un remplissage sémantique qui ne peut trouver son essence que vers la droite, siège de l'information nouvelle. Cette caractéristique sémantique de when vient alimenter mon propos.

En effet, dans le cas qui me préoccupe, when indique un contraste, il met par conséquent [he - marry her] en valeur, en tant que contenu informationnel venant le remplir sémantiquement. Et l'on s'aperçoit alors d'une chose étonnante: la pose même de ce repère en when indique implicitement qu'à partir de ce moment-là, que depuis ce moment-là, la situation a changé. Tout se passe comme si when mettait en place deux intervalles, l'un le précédant $\left(T_{3}-T_{2}\right)$ et l'autre lui succédant $\left(T_{2}-T_{1}\right)$. De plus, la pose $d u$ second intervalle va de pair avec l'instauration d'une nouvelle relation (nous passons de la continuité à la rupture). Le fait que ce que when met en place soit deux intervalles est très intéressant. On peut aller jusqu'à dire que son comportement s'apparente alors en effet à celui de since et $f r^{16}$. Mon énoncé signifie essentiellement deux choses, même tacitement, qui sont :

- She had been healthy and beautiful for + durée when he married her

- et She has not been ... since (he married her).

41 When permet ici de rendre compte des deux relations à la fois. Il n'a plus rien à voir avec after, before ou as vus plus haut ${ }^{17}$.

\section{Rôle de l'énonciation}

La réflexion peut être poursuivie en cherchant la raison pour laquelle c'est l'association when + prétérit qui va venir servir de repère à had been .

En fait, cela semble logique si le but recherché est la mise en exergue du contraste. Ce qui importe alors, c'est le tournant, le pivot, c'est-à-dire le mariage qui est à l'origine du bouleversement. En tant que tel, il insiste sur la rupture de la continuité entre "avant" ([she - be healthy...]) et "après" ([he - marry]). Les deux événements se situant dans un avant par rapport à $T_{0}$, qui est la situation d'énonciation repère par excellence, le repérage de [she - be healthy...] se fait ipso-facto également par rapport à $\mathrm{T}_{0}$. La rupture (invariant sémantique du prétérit) et le contraste se mettent donc en place à cause du triple repérage qui se fait à partir de $\mathrm{T}_{0}$.

44 A terme, l'association de had been et de when autorise à pratiquer la rupture suivie d'une ouverture à droite, car le past perfect vient souligner une caractéristique antérieure sous forme d'un état qui ne permet pas de conduire à une identité permanente, puisque cette 
caractéristique est soumise à repérage. L'emploi conjugué des deux constructions en anglais témoigne d'un enchaînement très fort entre les propositions. Il y a d'abord expression de la continuité puis accent sur le moment qui se trouve dire, à la fois, la clôture et l'ouverture. L'énoncé est très dialectique, il dit l'ouverture (avec had been) puis la clôture qui s'ensuit (avec when + married). Ce fonctionnement de la langue conduit à la réalisation d'une double opération que l'on ne retrouve pas en français ${ }^{18}$.

D'abord, when opère un bouclage à droite : il permet de revenir sur ce qui a été mais n'est plus. Il est la trace d'une anaphore (sa nature de subordonnant lui donne d'office le statut de rang second dans l'élaboration de la relation $\mathrm{S} / \mathrm{P}$, rang second qui suppose une étape initiale) avec retour sur une situation antérieure. Le bouclage présuppose en effet que l'on ne peut entamer quelque chose de nouveau dans le discours si l'on n'a pas circonscrit ce que l'on avait auparavant prévu de dire. Une entité se définit alors par ce qui lui est immédiatement consécutif ; et c'est précisément le cas dans mon énoncé.

Ensuite, il enchaîne sur la droite (rôle de wH-) pour donner la cause de la rupture. La chose se trouve à nouveau confirmée par les temps de chaque proposition. La suite past perfect / prétérit, (dans cet ordre), tend à impliquer que la proposition en when est temporellement indépendante de la matrice ${ }^{19}$. En effet, le prétérit sur [marry] ne peut se construire par rapport au past perfect sur be. C'est le past perfect qui, en tant que "temps relatif", se construit relativement à un prétérit, et le past perfect sur BE ne peut avoir été construit que relativement à un autre prétérit que celui sur [marry]. Soit, schématiquement :

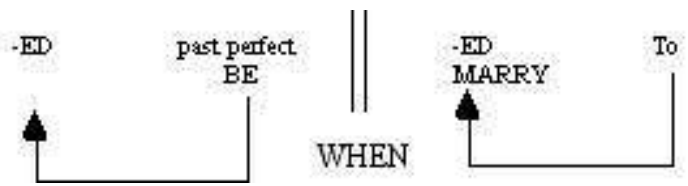

Ce qui n'est pas sans conséquences sur le cadre temporel auquel appartient when. A quel temps précisément renvoie when ? Quel est le temps de when? A priori, nous serions tentés de croire que when s'inscrit dans la sphère temporelle de référence, c'est-à-dire $T_{2}$ pour mon énoncé $(1)^{20}$. Il semblerait que ce ne soit pas le cas. When ne serait pas là pour poser le rapport au temps, la conjugaison sur les verbes s'en chargeant. When serait là pour relayer le rapport entre les relations prédicatives, lesquelles sont temporellement marquées. When ne ferait que définir un espace dans lequel les relations S/P viennent prendre place. Je pense que when n'intervient pas au même niveau que les autres constituants, qu'il n'a pas le même statut. Il fonctionnerait davantage comme un opérateur "extrapropositionnel"21 qui engloberait et les relations S/P et la situation de référence (qu'elle soit énonciative ou textuelle). Il serait posé en dernier. Il est en effet très important de tenir compte de tous les repères autour desquels se construit (1), et notamment $\mathrm{T}_{\mathrm{o}}$. Il y a bien repérage, implicite, à $\mathrm{T}_{\mathrm{o}}$. Car le moment même de la narration crée les enchaînements temporels. Had been laisse percer la subjectivité de l'énonciateur qui s'implique dans le repérage au regard de la référence, objective, qu'est [marry her]. La valeur du past perfect de (1) est métalinguistique. Chose logique puisqu'il s'agit d'une périphrase qui, par définition, va expliciter les relations entre constituants. A savoir ici l' analyse et l'explication, contrairement à la valeur synthétique du prétérit simple. Nous aurions donc là une présentation de when dans un fonctionnement lié à $\mathrm{T}_{\mathrm{o}}$.

Nous sommes alors en droit de nous demander si when tient le rôle qui est le sien en (1) précisément parce qu'il est posé en $\mathrm{T}_{\mathrm{o}}$ et non au moment $\mathrm{T}$ de, ou décrit par, l'énoncé. 
Quelle que soit la nature exacte de when en effet, il n'en est pas moins un mot en wH- où wH- est, nous le savons, le signe d'un déficit informationnel. Mais il est dans le même temps présupposant. Il présuppose l'existence de cette information, qui va être d'ordre temporel.

Dire :

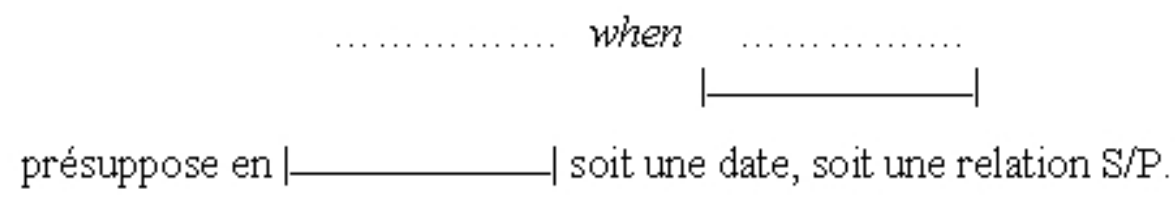

Poser when revient implicitement à annoncer quelque chose à sa suite qui va permettre d'identifier de façon certaine le moment en question. When instaure ipso-facto le paramètre nécessaire à la localisation, dans l'espace "temps", de la principale. Dans l'absolu, le "circonstant temps" fait partie intégrante, à tout le moins théoriquement, de toute mise en relation d'un sujet et d'un prédicat. C'est, entre autres, de cet état de faits dont when serait la preuve. Car parallèlement, when présuppose également son rattachement à l'information de la matrice, au "then" qu'il contient. Ce caractère éminemment présupposant de when expliquerait pourquoi, en (1), il n'établirait pas une hiérarchisation temporelle entre les deux propositions mais serait le signe de sa préconstruction. When participerait alors plus de la synthèse que de l'analyse. Ou plutôt, il synthétiserait après analyse. Il synthétiserait l'existence du rapport implicite au temps ($\mathrm{EN}$ ), sa matérialisation en un repère précis pour la relation $\mathrm{S} / \mathrm{P}$ donnée dans la subordonné (wн-) et le rattachement notionnel à la matrice sans laquelle la subordonnée n'a pas de raison d'être (тH-). Dire when nécessite la construction au préalable (тн-) de deux situations ${ }^{22}$ sur lesquelles il s'appuie notionnellement, à part égale. When regarde alors à gauche et à droite, et non pas dans une seule direction. C'est tout ce travail de préparation intellectuelle dont wH-en serait la trace. Le temps de when serait tout notionnel, et se calculerait depuis $T_{0}$. When serait la dernière pierre de l'édifice propositionnel reconstruit en $\mathrm{T}_{\mathrm{o}}$ par l'énonciateur. Il serait le "témoin $\mathrm{T}_{\mathrm{o}}$ " de toute cette reconstruction mentale de l'organisation et de l'enchaînement des événements décrits dans les propositions. Schématiquement, le phénomène se représenterait ainsi :

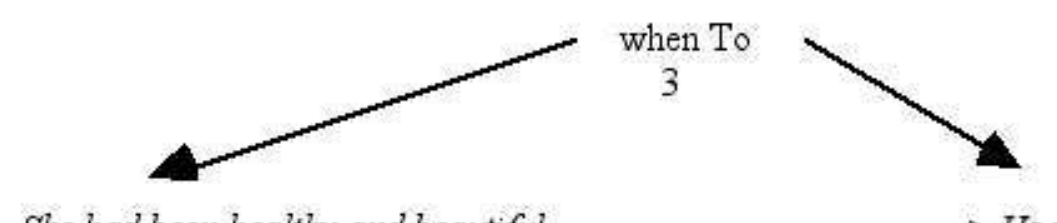

She had been healthy and beautiful

He married her:

1

2

51

When ne serait pas, en soi, temporel mais, venant clore la prédication, il serait là pour indiquer une lecture d'ordre temporel à l'énoncé. Il renseignerait sur le fait que la relation entre les événements décrits dans les différentes propositions de l'énoncé serait une question de temps (et non de lieu ou de manière, par exemple). ${ }^{23}$ Comment ceci serait-il possible? Et bien, nous savons que when est un outil linguistique utilisé par la langue pour symboliser le rapport au temps. When n'apparait cependant jamais seul ${ }^{24}$ : il est toujours accompagné de verbes porteurs de la marque du temps. Or ce repérage temporel, à partir de quel point de référence se crée-t-il sinon $\mathrm{T}_{\mathrm{o}}$ ? Il n'y aurait donc, en 
réalité, rien d'exceptionnel à ce que when soit posé depuis $\mathrm{T}_{\mathrm{o}}$. Il témoignerait de toute la préparation inconsciente de l'acte de discours.

\section{Conclusion}

She had been healthy and beautiful when he married her illustre un cas extrême dans lequel la conjonction de divers paramètres (sujet, temps, aspect, types de procès, logique...) ne répond à aucune formation communément répertoriée et autorisée, mais est néanmoins grammaticale. Ce problème semble insoluble s'il est abordé dans une optique traditionnelle qui tend à vouloir attribuer un rôle précis (et définitif) à chacun des constituants d'un énoncé. En revanche, si l'on est prêt à admettre que les constituants peuvent évoluer sémantiquement, ou que tel paramètre présent dans leur sémantisme peut être sélectionné prioritairement à un autre, alors rien ne s'oppose à ce que l'on adopte une vision plus globale, plus globalisante, concernant l'interaction de ces constituants. Quelques éléments de réponse peuvent alors être apportés.

Il en est ainsi pour when. De son invariant qui repose sur l'expression d'un déficit informationnel, d'une quête d'un sémantisme (wH-) et sur le rattachement métaphorique au temps (-EN), il tire bien la possibilité de fonctionner comme relateur entre deux relations S/P. L'observation de la langue nous a indiqué ici que la nature de cette relation posée par when peut varier en profondeur malgré une similitude de comportement en surface ${ }^{25}$. J'ai tenté de montrer comment se crée la "polyvalence contextuelle" de when, en m'appuyant sur un énoncé dont les formes verbales viennent illustrer un (le) fonctionnement secret de when.

Et un énoncé apparemment anodin de témoigner, une nouvelle fois, qu'il est vain de dissocier catégories grammaticales et productions en contextes.

\section{BIBLIOGRAPHIE}

BORILLO, Andrée, 1988. "Quelques remarques sur QUAND connecteur temporel." Langue Française, 77, 71-91.

BOUSCAREN, Janine, 1993. Cahiers de recherche en grammaire anglaise, T 6 : "Types de procès et repères temporels". Paris, Gap : Ophrys.

соTтE, Pierre, 1987. "Réflexions sur l'emploi des temps du passé en français et en anglais à la lumière de deux évolutions récentes du système verbal de l'anglais." Contrastes, 14-15, 89-161.

COTTE, Pierre, 1996. L'Explication grammaticale de textes anglais. Paris : PUF.

COTTE, Pierre, 1997. Grammaire linguistique. Paris : Didier Erudition, CNED.

DECLERCK, Renaat, 1996. "A functional typology of English When-clauses." Functions of Language, 3, $2,85-234$.

DECLERCK, Renaat, 1996. "Tense choice in adverbial when-clauses." Linguistics, 34, 225-261. 
DECLERCK , Renaat, 1997. When-clauses and Temporal Structure. Routledge: London.

DELECHELLE, Gérard, 1983. "Antériorité, simultanéité, concomitance et causalité en Anglais." Tréma , 8, 31-48.

DELECHELLE, Gérard, 1980. "When et l'opposition TH- / WH-" 1980, 29 p., (dactylographié).

DILYS, Rosalind, 2000. Les Subordonnants exprimant la concomitance en anglais contemporain : recherches sur when, as et while. $347 \mathrm{f}$. dactyl. Thèse. Université de Caen.

GUILLEMIN-FLESHER, Jacqueline, 1981. Syntaxe comparée du français et de l'anglais : problèmes de traduction. Paris, Gap : Ophrys.

HEINÄMÄKI, Orvokki, 1978. Semantics of English Temporal Connectives. Helsinki: Bloomington, Indiana University Linguistics Club.

LAPAIRE, Jean-Rémi et ROTGE, Wilfrid, 1991. Linguistique et grammaire de l'anglais. Toulouse : Presses Universitaires du Mirail.

LE GOFFIC, Pierre, 1986. Points de vue sur l'imparfait. Caen : Centre de Publications de l'Université de Caen.

LE GUAY, Emmanuelle, 1991. "Le Pluperfect." 150 f. dactyl. Mémoire de DEA : Théories et Analyses Linguistiques. Lille 3.

LE GUAY, Emmanuelle, 1995. Recherches sur le Past Perfect en anglais contemporain. $430 \mathrm{f}$. dactyl. Thèse. Université de Paris IV - Paris Sorbonne.

QUIRK, Randoplh et WRENN, Charles Leslie, 1995. An Old English Grammar. Londres / New York: Methuen.

QUIRK, Randolph, et al., 1985. A Comprehensive Grammar of the English Language. Londres : Longman. ROUSSEL, Emmanuelle, 1999. "She had been healthy and beautiful when he married her", Anglophonia: English Linguistics, 6. Toulouse : Presses Universitaires du Mirail, 235-258.

VISSER, F., 1963, 1966, 1973. "Syntactical Units with one Verb", "Syntactical Units with one Verb", "Syntactical Units with two Verbs and More" in An Historical Syntax of the English Language, Leiden: E.-J. Brill.

WEINRICH, Harald, 1973. Le Temps : le récit et le commentaire. Paris : Seuil.

WIERZBICKA, Anna, 1988. "Why can you have a drink when you can't *have an eat?" in: The Semantics of Grammar. Amsterdam: John Benjamins Publishing Company, 293-357.

\section{NOTES}

1. "Les différentes valeurs de when correspondent à des degrés divers de cohésion entre les deux propositions, qui résultent eux-mêmes de différences dans les opérations." (G. Deléchelle 1980 : 22).

2. G. Deléchelle 1983 : 45 . Le sens donné par l'auteur au terme "concomitant" est le suivant, qu'il emprunte à A. Culioli: "lorsqu'on repère deux énoncés l'un par rapport à l'autre, on a une relation qui est soit de concomitance, soit de consécution, soit composite (causalité)." Culioli, Antoine, (1976) Recherche en Linguistique. DRL Paris VII, p. 180 et Le Français Moderne. (4), octobre 1987, note 4, p. 303.

3. $C f$ E. Roussel 1999. Cet énoncé sera dorénavant désigné par (1). 
4. $\mathrm{T}_{1}$ désigne le repère par rapport auquel est évalué le past perfect, c'est-à-dire un moment du passé, distinct de l'énonciation qui elle est symbolisée par $\mathrm{T}_{\mathrm{o}}$.

5. Le sémantisme du past perfect, plus encore que pour d'autres constructions, est étroitement lié aux éléments contextuels à cause de chaque constituant qui entre dans sa composition (entre autres le prétérit sur HAVE et le perfect). La valeur de ces constituants se trouve actualisée par n'importe quel type de facteur, sans qu'il soit obligatoirement temporel ou aspectuel du reste. Un nom propre peut par exemple déclencher en priorité le renvoi au révolu (-ed) ou au perfect (haveen) selon son statut au moment choisi comme repère. Prenons Jim had written poems. Si Jim est mort au moment pris comme repère, la construction traduit une rupture référentielle par rapport à $\mathrm{T}_{1}$ (la relation prédicative n'est référentiellement plus vraie en $\mathrm{T}_{1}$ ). Même chose si Jim travaille sur un roman ou s'il a cessé cette activité. A cette équation s'ajoute à l'évidence l'aspect interne propre à chaque lexème verbal utilisé.

6. Comme dans: When he had gone, I cried. Ou When Tracy awakened in the morning, ... she ordered a light breakfast and hot, black coffee. (S. Sheldon, 1985). Enoncés empruntés à E. Zeitoun, p. 89 (Zeitoun, E., When et la temporalité. in BOUSCAREN, Janine, 1993. Cahiers de recherche en grammaire anglaise, $\mathrm{T} 6$ : "Types de procès et repères temporels". Paris, Gap : Ophrys.)

7. La création de ces énoncés et leur comparaison avec celui en when crée un lien direct entre cet article et l'excellente thèse que mon amie et collègue Rosalind Dilys a soutenue sur le sujet en décembre 2000. Je tiens cependant à souligner ici que j'ai commencé à penser cet article il y a environ dix ans, et l'ai rédigé à la fin des années 1990. D'où l'absence globale, qui peut paraître surprenante, de références au travail de R. Dilys. J'ai souhaité, par honnêteté intellectuelle, conserver à mon travail son état initial. Mais le lecteur est fortement incité à se reporter au travail de R. Dilys et notamment, au sujet de cet énoncé, p. 328.

8. Comme dans: He was smoking when she was watering the flowers. $v .{ }^{*}$ He was smoking when she smoked.

9. Nous savons que le prétérit simple en soi ne bloque aucunement un sémantisme de recouvrement. Ou en tout cas, que la seule conjugaison du verbe n'est pas un argument suffisant pour véhiculer le recouvrement. D'autres paramètres sont bien évidemment en jeu, dont notamment l'aspect interne des lexèmes verbaux en présence.

10. J.-R. Lapaire, W. Rotgé : 1991 : 242.

11. Le procédé me semble certes être un choix de l'énonciateur. Mais pour qui au juste ? On est en droit de se poser la question. Est-ce pour asseoir ses souhaits énonciatifs propres ou bien pour préparer une meilleure compréhension de l'énoncé chez son co-énonciateur?

12. D'où sa co-occurrence possible avec then, dont nous venons de voir qu'il est un autre signe d'un premier repérage : (3) She had been healthy and beautiful then.

13. Et toutes ses propriétés d'opérateur de localisation.

14. Représentation dans laquelle le past perfect reproduit ce qui se passe pour l'élaboration du prétérit (à savoir une conjugaison présente d'un fait passé) pour l'instant repère $\mathrm{T}_{1}$. C'est-à-dire que dans cette hypothèse, le past perfect refait un prétérit d'un present perfect initial (noté have Ved2) par rapport à $\mathrm{T}_{1}$. La représentation du prétérit étant quant à elle $V_{\text {ed1 }}$. D'où le système, dont le rapprochement entre les deux premiers termes est dû à P. Cotte, (1996: 73) :

- prétérit S Ved1 prétérit

- Present perfect S Have Ved2 participe passé

- past perfect S [Have Ved2] ed3

15. Et je pense que bien qu'il apparaisse avant -ed2 dans la chaîne linéaire d'écriture du past perfect (had $V$-en), il serait en réalité posé en dernier et viendrait valider la préconstruction de l'acquis notionnel véhiculé par le parfait. L'hypothèse est développée dans E. Roussel 1999.

16. Dans leur emploi prépositionnel et non pas conjonctif. Je ne prétends pas poser when comme équivalent de since et for en règle générale. Il n'est question ici que de comportement. Même remarque à propos de until. 
17. Ni encore whenever ou while bien sûr.

18. Dans l'énoncé obtenu lors de la traduction : "Elle était belle et bien portante quand il l'épousa / l'avait épousée", on assiste (avec le passé simple ou le plus-que-parfait) à l'annonce du décalage temporel qui va venir expliciter la cassure. L'important alors n'est plus le pivot, mais la chronologie.

19. Si l'on s'en réfère à R. Declerck (1997: 227): "That it [shifting the domain] is possible in narrative WCs [when-clauses] is another indication that the latter behave like independent clauses rather than like canonical WCs."

20. R. Declerck (1997: 44) mentionne le fait: "... the time of the when-clause situation is not related directly to the time of the head-clause situation or to the time of speech. The times of the two situations are related to each other indirectly through being included in a common interval defined by when."

21. Ou "interpropositionnel " ? Il parait difficile de trancher entre les deux appellations.

22. Deux, et non une seule, car when est le lien entre deux $\mathrm{S} / \mathrm{P}$, il ne peut suffire à repérer une seule S/P. Cf. (3a) * She had been healthy and beautiful when.

23. Il faut préciser que ce ne sont pas tant les événements qui sont repérés depuis $\mathrm{T}_{\mathrm{o}}$ que les moments de validation des prédications qu'ils dénotent.

24. Sauf énoncé elliptique, auquel cas les informations nécessaires sont préconstruites. Ce qui revient au même.

25. CfR. Dilys, p. 54.

\section{RÉSUMÉS}

L'objectif de cet article est de montrer la manière dont when fonctionne dans un exemple précis. L'étude du marqueur porte sur un seul énoncé. Il s'avère que le sémantisme de when est complètement différent de celui qu'il est susceptible d'avoir dans ses autres emplois avérés. Dans notre cas, il n'est plus question de connections d'ordre temporel que l'on pourrait gloser à l'aide de after, as, before, while ou encore whenever. De façon surprenante, ce sont les temps utilisés sur chacun des verbes des propositions de notre énoncé qui font que when y a cette valeur spécifique. Valeur qui pourrait tout à fait naître du moment même de l'énonciation.

This paper sets out to show how the marker when works in a specific example. The marker is here studied within one single utterance. It turns out that the meaning taken on by when is completely different form the one it is liable to have in all its accepted uses. In our case there is no further question of connections of a temporal nature we could express with after, as, before, while or even whenever. What is surprising then is that it is the tenses on the verbs in each clause making up our utterance that endow when with its specific value. A value which may well be built from the moment of utterance.

\section{INDEX}

Mots-clés : when, préconstruction, rattachement métaphorique, temps notionnel, To, relateur, polyvalence 


\section{AUTEUR}

EMMANUELLE ROUSSEL

Université de Caen - CRISCO (UMR 6170) 